\title{
Residual and recurrent shunts after implantation of Cook detachable duct occlusion coils
}

\author{
O Uzun, D Dickinson, J Parsons, J L Gibbs
}

Department of

Paediatric Cardiology, Killingbeck Hospital, York Road, Leeds LS14 6UQ, UK

O Uzun

D Dickinson

J Parsons

J L Gibbs

Correspondence to: Dr Gibbs.

Accepted for publication 24 November 1997

\begin{abstract}
Objective-To assess the presence and outcome of Doppler detectable shunts following implantation of the Cook detachable PDA coil.

Design-Prospective study.

Setting-Tertiary paediatric cardiac centre.

Patients-76 consecutive patients undergoing coil implantation (80 procedures).

Main outcome measures-Detection and colour Doppler echocardiographic appearance of residual or recurrent shunts, the timing of the appearance of recurrent shunts, and the time taken for spontaneous resolution of these shunts.

Results-Immediate occlusion was achieved in 52 patients. At one month 63 patients had complete occlusion and after three months the duct was completely occluded in 67 patients. In 27 cases small residual shunts were detected on echocardiography 10 minutes after the completion of the implantation procedure; 15 of these had resolved by 24 hours and 20 had resolved by three months. Recurrent shunts were detected after apparent initial complete occlusion in 11 cases 24 hours after coil implantation and in two cases one month after the procedure. Six recurrent shunts resolved on later follow up. Residual shunts appeared as single jets after implantation of a single coil, but up to three separate jets were detected after implantation of multiple coils.

Conclusions-Spontaneous resolution of small residual shunts occurs in most
\end{abstract}

patients. The recurrence of small shunts after apparent complete occlusion suggests that recanalisation of the duct may occur in a small percentage of patients up to one month after occlusion. Residual shunts may take the form of multiple residual jets that may require implantation of further coils to achieve complete duct occlusion.

(Heart 1998;79:220-222)

Keywords: coil occlusion; arterial duct; congenital heart disease

In Europe the Cook detachable PDA coil has become one of the most popular devices for closing the arterial duct. It is easy to deliver and more than one device may be implanted during the same catheter procedure. Its cost is approximately a 10th that of the Rashkind umbrella and early studies have suggested better results than with the Rashkind devicecomplete occlusion in about $90 \%$ of cases after a single procedure. However, only short term results have been reported. ${ }^{12}$ This report, part of a large prospective study of duct occlusion, describes the incidence and appearance of residual shunts after coil implantation and describes the new observation of recurrent shunting after initial complete occlusion.

\section{Patients and methods}

Eighty procedures were performed in 76 patients. Venous access alone was used in most patients. After an aortogram (via the duct) coils were implanted using either a single $4 \mathrm{~F}$ venous catheter or, in cases where a single coil
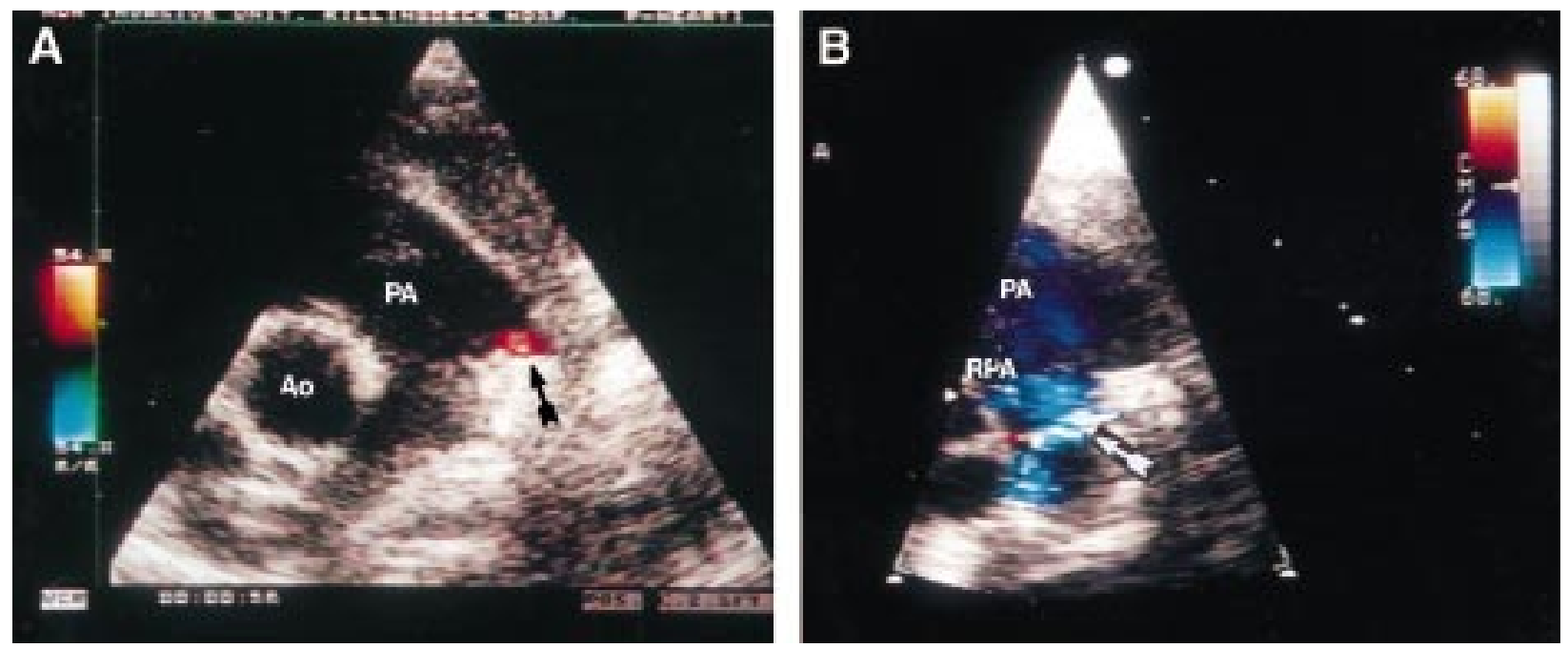

Figure 1 Single residual ductal jets in two patients with a single coil implanted. (A) A single jet is seen at the superior margin of the coil; (B) the residual jet is at the inferior margin with the jet directed towards the origin of the right pulmonary artery (RPA). Ao, aorta; PA, pulmonary artery. 


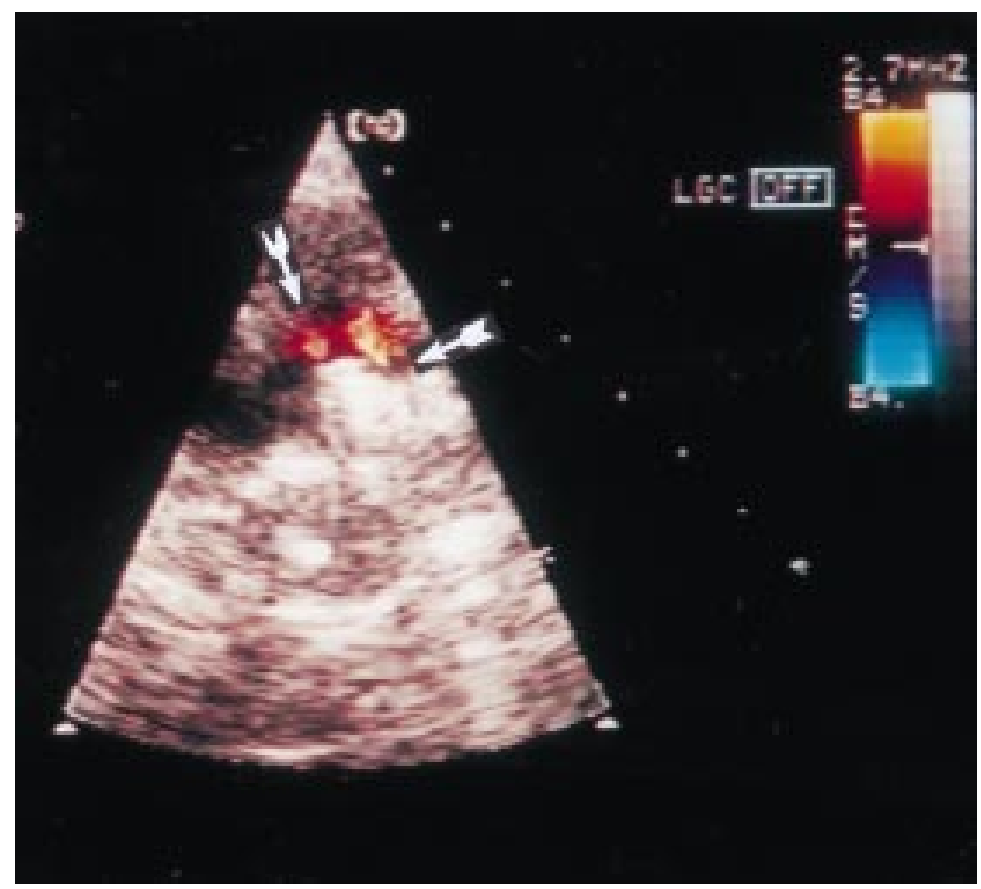

Figure 2 Two separate jets seen in the pulmonary trunk after implantation of two $5 \times 5$ coils, one arising at the superior margin of the coil and the other at the centre of the coil (arrowed).

appeared unstable, two coils were deployed simultaneously through two $4 \mathrm{~F}$ catheters.

Single coils were implanted in 58 cases, two coils in 18, and three coils in 4 cases. Echocardiography was performed at the end of the procedure (10 minutes after coil implantation) in the catheter laboratory and repeated the following morning, at one, three, and six months, and one year later if possible.

\section{Results}

COMPLETE OCCLUSION

Complete occlusion of the arterial duct occurred in 53 of the 80 procedures $(66 \%)$ immediately after coil implantation, in $72 \%$ of cases by 24 hours, in $82 \%$ at one month, and $88 \%$ at three months. The immediate occlu- sion rate was $80 \%$ when a single coil was used but only $30 \%$ when multiple coils were used, although the complete closure rate at late follow up was similar in the two groups $(90 \%$ and $85 \%$, respectively).

\section{RESIDUAL SHUNTS}

Small residual shunts (which we thought may close spontaneously) were detectable by colour flow Doppler in the 27 cases. Fifteen (56\%) of these residual shunts had disappeared by the following morning, $70 \%$ had resolved by one month, and $74 \%$ by three months. Spontaneous closure did not occur later than three months after coil implantation. Resolution of residual shunting was as likely to occur with a single coil as with multiple coils.

\section{RECURRENT SHUNTS}

In the 53 cases where the duct appeared to be completely occluded in the catheter laboratory small shunts were detectable by colour flow Doppler the following morning in 11 cases, and in two cases a new shunt appeared one month after coil implantation. The latter two shunts were caused by device embolisation, in the other 11 patients there was no evidence of device movement. Six of these 11 recurrent shunts resolved spontaneously on later follow up but small shunts remain in the other five. Recurrent shunts appeared as likely to close spontaneously as did residual shunts, regardless of the number of coils implanted.

SHUNT JET MORPHOLOGY: SINGLE COIL

When a residual or recurrent shunt was detected after a single coil was implanted, only a single jet was seen on colour flow Doppler (fig 1). In contrast to residual jets after implantation of the Rashkind umbrella (when the jet almost always occurs at the superior margin of the device), jet position and direction was very variable after coil implantation, sometimes occurring at the superior margin, sometimes at the inferior margin (directed towards the right pulmonary artery), and sometimes through the
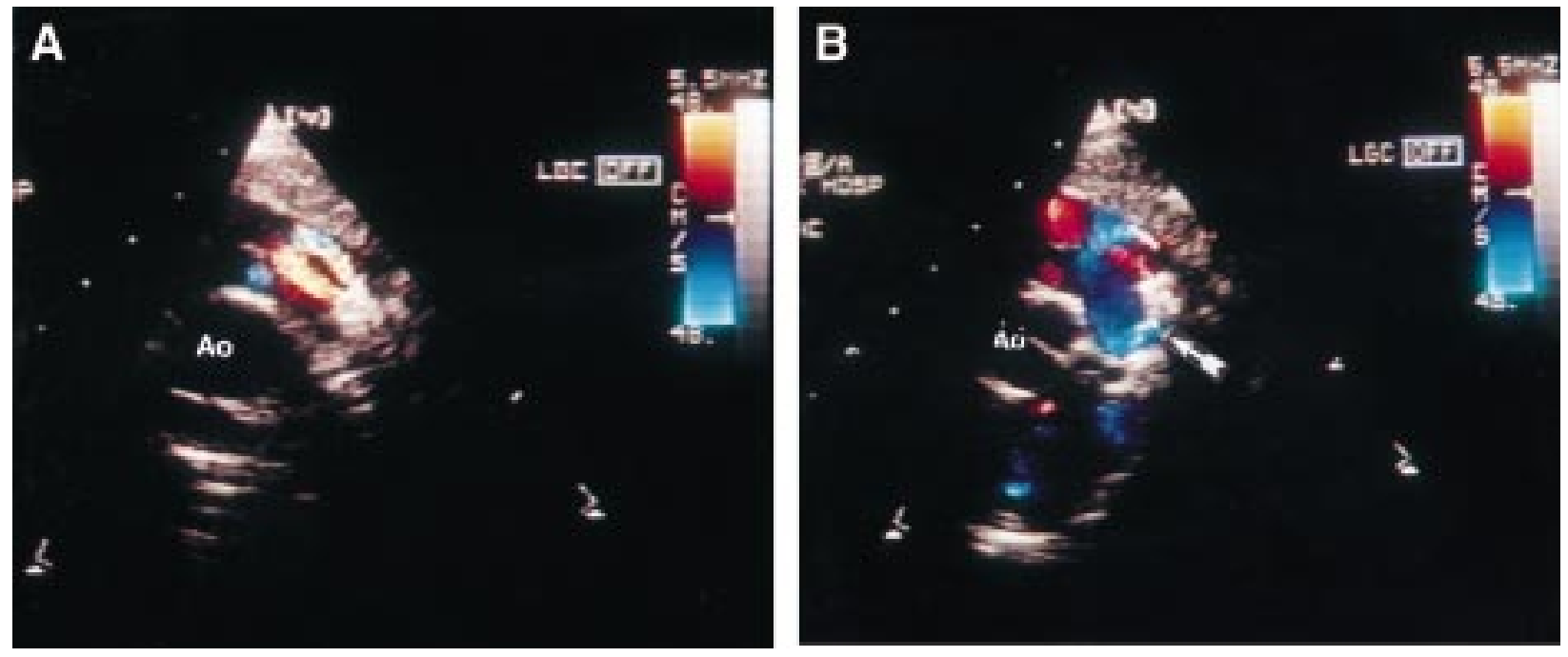

Figure 3 In this patient with three coils there initially appeared to be two residual shunt jets (A), but angulation of the transducer (B) revealed a third jet directed towards the right pulmonary artery (arrowed). Further intervention was required because of haemolysis, and three further coils were needed to completely occlude the residual shunt. Ao, aorta. 
centre of the coil. No particular relation between coil orientation and jet morphology was apparent.

SHUNT JET MORPHOLOGY: MULTIPLE COILS Two of the four patients who had residual shunts after multiple coil implantation had multiple shunt jets. Two jets were detected (at the superior margin and through the centre of the coil) in one patient (fig 2) and three separate jets (superior, central, and inferior) were detected in the other patient (fig 3 ). The patient with three jets developed haemolysis and each jet required a separate additional coil to achieve complete duct occlusion.

\section{Discussion}

Experience with duct occlusion using the Rashkind umbrella suggested that early residual shunts occurred in up to $85 \%$ of patients, that the residual shunt usually took the form of a single jet at the superior margin of the device, and that spontaneous resolution occurred in approximately $79 \%$ of cases. ${ }^{1}$ Ducts occluded using detachable coils clearly do not behave in the same manner. Our policy of using a single coil unless the duct was large resulted in an immediate residual shunt in 34\% of cases. This figure may well have been reduced by a more aggressive policy of multiple coil implantation, but probably at the cost of an increased risk of complications such as left pulmonary artery stenosis. After a single coil has been implanted the residual shunt takes the form of a single jet that may occur at the superior rim of the coil, at the inferior rim (when it may be directed towards the origin of the right pulmonary artery and may be easily missed), or through the centre of the coil. When more than one device is implanted more than one residual jet may occur and this may have important implications if further intervention is contemplated.

Our findings suggest that $74 \%$ of residual shunts will resolve spontaneously within six months, but we were disappointed to find that in $21 \%$ of patients ductal shunts were detected on follow up after early echocardiography suggested complete occlusion. That recurrent shunts were detected in two cases more than a month after coil implantation suggests that these recurrent shunts are most likely to result from genuine recanalisation (which may occur after coil embolisation of other vessels ${ }^{3}$ ) rather than from minor degrees of device migration or reversal of transient mechanically induced ductal spasm. ${ }^{4}$ As careful echocardiographic studies were performed as part of a prospective study it seems very unlikely that small residual shunts were simply missed by the echocardiographer early after coil implantation. Recurrent shunts appear to be as likely to resolve spontaneously as residual shunts, but in some cases small residual shunts will persist and may require further coil implantation to achieve complete duct occlusion.

1 Uzun O, Hancock S, Parsons JM, et al. Transcatheter occluUzun O, Hancock S, Parsons JM, et al. Transcatheter occlusion of the arterial duct with Cook
experience. Heart 1996;76:269-73.

2 Tometzki AJP, Arnold R, Peart I, et al. Transcatheter occluTometzki AJP, Arnold R, Peart I, et al. Transcatheter occlu-
sion of the patent ductus arteriosus with Cook detachable coils. Heart 1996;76:531-5.

3 Perry SB, Radtke V, Fellows KE, et al. Coil embolisation to occlude aortopulmonary collateral vessels and shunts in patients with congenital heart disease. F Am Coll Cardiol 1989;13:100-8.

4 Shim D, Fedderly RT, Beekman RH III, et al. Follow-up coil occlusion of patent ductus arteriosus. $7 \mathrm{Am}$ Coll Cardiol 